spectively. $\rho$ and $M$ are the density and molecular weight of water, respectively. $\Delta G_{\text {cycle }}$ is the molar Gibbs free-energy change of absorbed water during one expansion/contraction cycle. $f_{\text {ad }}$ is the adhesive force coefficient between PEE-PPy films and moist substrates. Given a certain $E, R$, and $f_{\text {ad, }}$ Eqs. 1 and 2 roughly define a theoretical maximum and minimum limit on the required thickness of the actuator to perform fast locomotion. In practice, we found that the optimal thickness for PEE-PPy actuators on a moist paper was roughly 15 to $40 \mu \mathrm{m}$. Actuators thinner than $15 \mu \mathrm{m}$ tended to stick to the moist paper, whereas actuators thicker than $40 \mu \mathrm{m}$ showed significantly slower locomotion.

Because this PEE-PPy actuator could continuously extract chemical potential energy out of ambient water gradients to perform mechanical work, it should be able to drive a piezoelectric element to convert the mechanical energy into electrical energy. A 9- $\mu$ m-thick piezoelectric polyvinylidene difluoride (PVDF) film was metallized, wired, and insulated on both faces (Fig. 4A). A $27-\mu \mathrm{m}$-thick PEE-PPy actuator was attached to one face of the PVDF element. When placed on a moist substrate with the actuator facing down, the actuator bent and stretched the PVDF element repeatedly (movie S5), generating an open-circuit voltage up to $3 \mathrm{~V}$. A 10 -megohm resistor was loaded onto this generator (Fig. 4B), and the peak output reached $\sim 1.0 \mathrm{~V}$ (Fig. 4D). Analysis indicated that the frequency of the alternating voltage signal was $\sim 0.3 \mathrm{~Hz}$ (fig. S12), which matched the motion frequency of the generator (movie S5). The average power output was $5.6 \mathrm{nW}$ (fig. S13), which corresponded to a power density of $56 \mu \mathrm{W} / \mathrm{kg}$ for the 100-mg generator. In contrast, the same PVDF element did not move on the moist substrate, and the recording showed only noise (fig. S14), with analysis of this background noise giving an average power output of $0.015 \mathrm{nW}$ (fig. S15). The generated alternating electrical pulses by the generator were rectified using a commercial full-wave bridge rectifier, then stored in a $2.2 \mu \mathrm{F}$ capacitor (Fig. 4C). Within 7 min of charging, the voltage of the capacitor was saturated to $\sim 0.66 \mathrm{~V}$ (Fig. 4E). This was lower than the peak output voltage of the generator, possibly due to voltage drop across the rectifying diodes and/or current leakage of the capacitor.

This PEE-PPy polymer composite system features an interpenetrating network of a rigid polymer with a soft, hydrolytically sensitive polymer that can perform water-gradient-induced displacement, converting the chemical potential energy in water gradients to mechanical work. Besides mechanical vibration energy, the generator based on this powerful actuator can use ubiquitous lowtemperature water gradients as its energy source, in contrast to state-of-the-art piezoelectric energy scavengers that rely solely on mechanical vibration energy (26). Thus, the water-gradient-driven actuator and generator demonstrated potential applications as sensors, switches, and power sources for ultralow-power devices.

\section{References and Notes:}

1. S. Minko, Responsive Polymer Materials: Design and Applications (Blackwell, Ames, IA, 2006).

2. E. W. H. Jager, E. Smela, O. Inganäs, Science 290, 1540 (2000).

3. Y. Osada, H. Okuzaki, H. Hori, Nature 355, 242 (1992).

4. A. Lendlein, H. Y. Jiang, O. Jünger, R. Langer, Nature 434, 879 (2005).

5. A. Lendlein, R. Langer, Science 296, 1673 (2002).

6. M. Camacho-Lopez, H. Finkelmann, P. Palffy-Muhoray, M. Shelley, Nat. Mater. 3, 307 (2004).

7. C. L. van Oosten, C. W. M. Bastiaansen, D. J. Broer, Nat. Mater. 8, 677 (2009).

8. Y. Yu, M. Nakano, T. Ikeda, Nature 425, 145 (2003).

9. J. Kim, J. A. Hanna, M. Byun, C. D. Santangelo, R. C. Hayward, Science 335, 1201 (2012).

10. A. Sidorenko, T. Krupenkin, A. Taylor, P. Fratzl, J. Aizenberg, Science 315, 487 (2007).

11. D. J. Beebe et al., Nature 404, 588 (2000).
12. G. H. Chen, A. S. Hoffman, Nature 373, 49 (1995).

13. J. M. Benns, J. S. Choi, R. I. Mahato, J. S. Park, S. W. Kim, Bioconjug. Chem. 11, 637 (2000).

14. M. E. Caldorera-Moore, W. B. Liechty, N. A. Peppas, Acc. Chem. Res. 44, 1061 (2011).

15. P. Fratzl, F. G. Barth, Nature 462, 442 (2009).

16. E. Smela, Adv. Mater. 15, 481 (2003).

17. R. H. Baughman, Science 308, 63 (2005).

18. H. Okuzaki, T. Kuwabara, T. Kunugi, J. Polym. Sci. B Polym. Phys. 36, 2237 (1998).

19. T. Ushiki, Arch. Histol. Cytol. 65, 109 (2002).

20. M. Shibayama, M. Sato, Y. Kimura, H. Fujiwara, S. Nomura, Polymer (Guildf.) 29, 336 (1988).

21. T. Park, S. C. Zimmerman, J. Am. Chem. Soc. 128, 13986 (2006).

22. X. M. Ren, P. G. Pickup, J. Phys. Chem. 97, 5356 (1993).

23. M. Szafran, Z. Degaszafran, J. Mol. Struct. 321, 57 (1994).

24. S. Hara, T. Zama, W. Takashima, K. Kaneto, Polym. J. 36, 151 (2004).

25. J. D. Madden, P. G. Madden, P. A. Anquetil, I. W. Hunter, in Electroactive Polymers and Rapid Prototyping (Materials Research Society, Warrendale, PA, 2002), vol. 698, p. 137.

26. Y. Qin, X. Wang, Z. L. Wang, Nature 451, 809 (2008).

Acknowledgments: M.M. and R.L. conceived the idea and designed the experiments. M.M. and L.G. performed the experiments. M.M., L.G., D.G.A., and R.L. contributed materials and/or tools, analyzed the data, and wrote the paper. This research was supported in part by a National Heart, Lung, and Blood Institute Program of Excellence in Nanotechnology (PEN) Award, contract no. HHSN268201000045C; National Cancer Institute grant CA151884; and Armed Forces Institute of Regenerative Medicine Award no. W81XWH-08-2-0034. Experimental procedures, additional data for materials characterization, and the device test are presented in the supplementary materials. We thank D. Bong for thoughtful discussion and $\mathrm{N}$. Zhang for help in the preparation of figures.

\section{Supplementary Materials}

www.sciencemag.org/cgi/content/full/339/6116/186/DC1

Materials and Methods

Supplementary Text

Figs. S1 to S16

Tables $\mathrm{S} 1$ and $\mathrm{S2}$

Eqs. $\mathrm{S} 1$ to $\mathrm{S3}$

Movies $\mathrm{S} 1$ to $\mathrm{S} 5$

References (27-32)

17 September 2012; accepted 9 November 2012 10.1126/science. 1230262

\title{
Sequence-Specific Peptide Synthesis by an Artificial Small-Molecule Machine
}

\section{Bartosz Lewandowski, ${ }^{1}$ Guillaume De Bo, ${ }^{1}$ John W. Ward, ${ }^{1}$ Marcus Papmeyer, ${ }^{1}$ Sonja Kuschel, ${ }^{1}$ María J. Aldegunde, ${ }^{2}$ Philipp M. E. Gramlich, ${ }^{2}$ Dominik Heckmann, ${ }^{2}$ Stephen M. Goldup, ${ }^{2}$ Daniel M. D'Souza, ${ }^{2}$ Antony E. Fernandes, ${ }^{2}$ David A. Leigh ${ }^{1,2 *}$}

The ribosome builds proteins by joining together amino acids in an order determined by messenger RNA. Here, we report on the design, synthesis, and operation of an artificial small-molecule machine that travels along a molecular strand, picking up amino acids that block its path, to synthesize a peptide in a sequence-specific manner. The chemical structure is based on a rotaxane, a molecular ring threaded onto a molecular axle. The ring carries a thiolate group that iteratively removes amino acids in order from the strand and transfers them to a peptide-elongation site through native chemical ligation. The synthesis is demonstrated with $\sim 10^{18}$ molecular machines acting in parallel; this process generates milligram quantities of a peptide with a single sequence confirmed by tandem mass spectrometry.

$\mathrm{C}$ ells achieve the sequence-specific synthesis of information-rich oligomers and polymers through the operation of complex molecular machines that transcribe information from the genetic code (1). The most extraordinary of these is the ribosome (2-4), a $\sim 2.6-\mathrm{MD}$ (bacterial) to 4.3-MD (eukaryotic) molecular machine found in all living cells that assembles amino acids from tRNA building blocks into a peptide chain with an order defined by the sequence of the mRNA strand that it moves along. Artificial small-molecule machines (5) have previously been used to store information $(6,7)$ and do mechanical work $(8-11)$; others have been employed in synthesis to processively epoxidize an unsaturated polymer $(12,13)$, switch "on" and "off" catalytic activity (14-17), and change the handedness of a reaction product (18). Large synthetic DNA molecules have been used to guide the formation of bonds between unnatural building blocks (19-22) and assemble

${ }^{1}$ School of Chemistry, University of Manchester, Oxford Road, Manchester M13 9PL, UK. ' ${ }^{2}$ chool of Chemistry, University of Edinburgh, The King's Buildings, West Mains Road, Edinburgh EH9 3]J, UK.

${ }^{*}$ To whom correspondence should be addressed. E-mail: david.leigh@manchester.ac.uk 
gold nanoparticles in particular sequences (23). Here, we report on the design, synthesis, and operation of a rotaxane-based small-molecule machine in which a functionalized macrocycle operates on a thread containing building blocks in a predetermined order to achieve sequencespecific peptide synthesis. The design of the ar- tificial molecular machine is based on several elements that have analogs in either ribosomal (2-4) or nonribosomal (24) protein synthesis: Reactive building blocks (the role played by tRNA-bound amino acids) are delivered in a sequence determined by a molecular strand (the role played by mRNA). A macrocycle ensures pro- cessivity during the machine's operation (reminiscent of the way that subunits of the ribosome clamp the mRNA strand) and bears a catalyst - a tethered thiol group - that detaches the amino acid building blocks from the strand and passes them on to another site at which the resulting peptide oligomer is elongated in a single specific

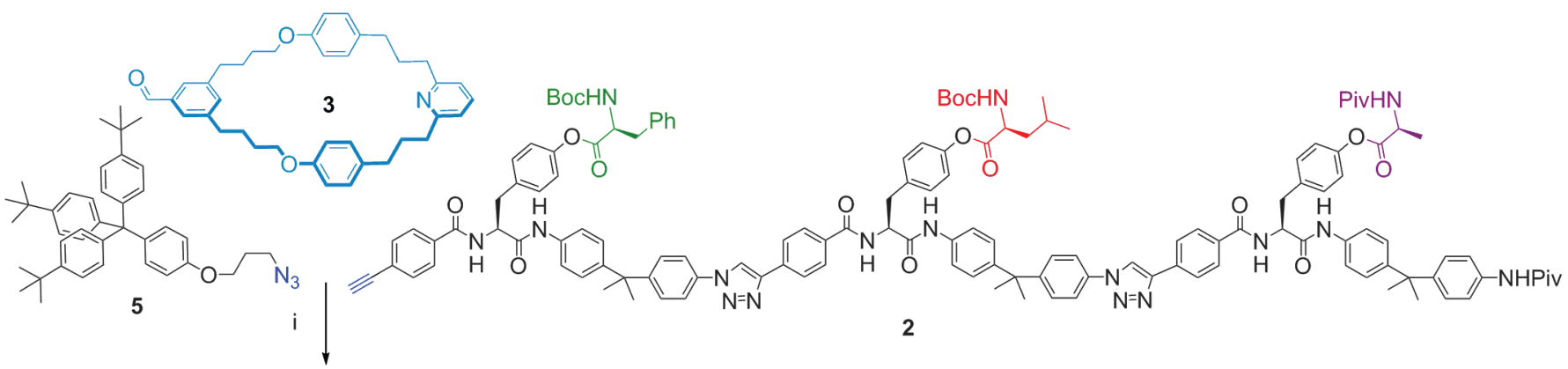

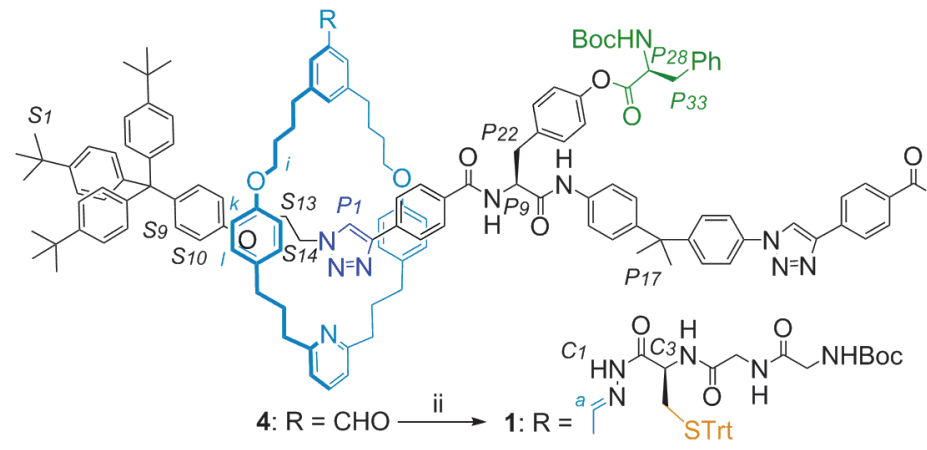

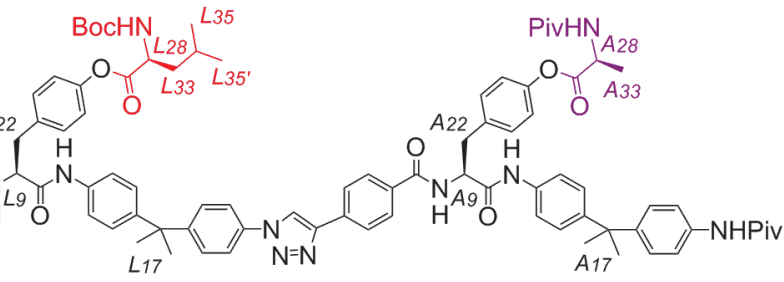

Fig. 1. Synthesis of rotaxane-based molecular machine $\mathbf{1}$, incorporating a strand bearing amino acid building blocks (2), a macrocycle (3) with a site for attachment of the reactive arm, and a terminal blocking group (5) that prevents the threaded macrocycle from coming off the strand until all of the amino acid groups have been cleaved. Structure $\mathbf{1}$ is shown as the major diastereomer; a small amount of epimerization $(<5 \%)$ of some of the acyl amino units occurs during incorporation into the track. Boc, $\mathrm{CO}_{2} \mathrm{C}\left(\mathrm{CH}_{3}\right)_{3} ; \mathrm{Piv}_{1}, \mathrm{COC}\left(\mathrm{CH}_{3}\right)_{3 i}$; Trt, $\mathrm{CPh}_{3}$; Ph, phenyl. Reaction conditions: (i) $\left.\mathrm{Cu}_{(\mathrm{CH}} \mathrm{CN}\right)_{4} \mathrm{PF}_{6}$ in dichloromethane:t-butanol (2:1), room temperature, 4 days, $30 \%$. (ii) $\mathrm{PhNH}_{2}$ (catalyst), BocGlyGlyCys(S-Trt) NHN=CHC $6 \mathrm{H}_{4} \mathrm{OCH}_{3}$ in 3:1 dimethylsulfoxide: aqueous 2-(N-morpholino)ethanesulfonic acid buffer (pH 6.0), $60^{\circ} \mathrm{C}, 2$ days, $90 \%$. The italicized letters indicate key signals in the ${ }^{1}$ H NMR spectrum shown in Fig. 2B. For the full lettering scheme and assignments, see the supplementary materials, page S28 (26).

Fig. 2. Proton NMR spectrum of (A) the noninterlocked thread and (B) rotaxane $\mathbf{1}$, in $d_{6}$-dimethylsulfoxide $(500 \mathrm{MHz}, 298 \mathrm{~K})$. Rotaxane 1 exists in both $E$ - and Z-hydrazone forms. The assignments correspond to the lettering shown in Fig. 1. C', S-Trt-cysteine; $G^{\prime}$, $\mathrm{N}$-Boc-glycine; $\mathrm{F}^{\prime}, \mathrm{N}$-Boc-phenylalanine; $\mathrm{L}^{\prime}, \mathrm{N}$-Bocleucine; $A^{\prime}, N$-Piv-alanine. ppm, parts per million.

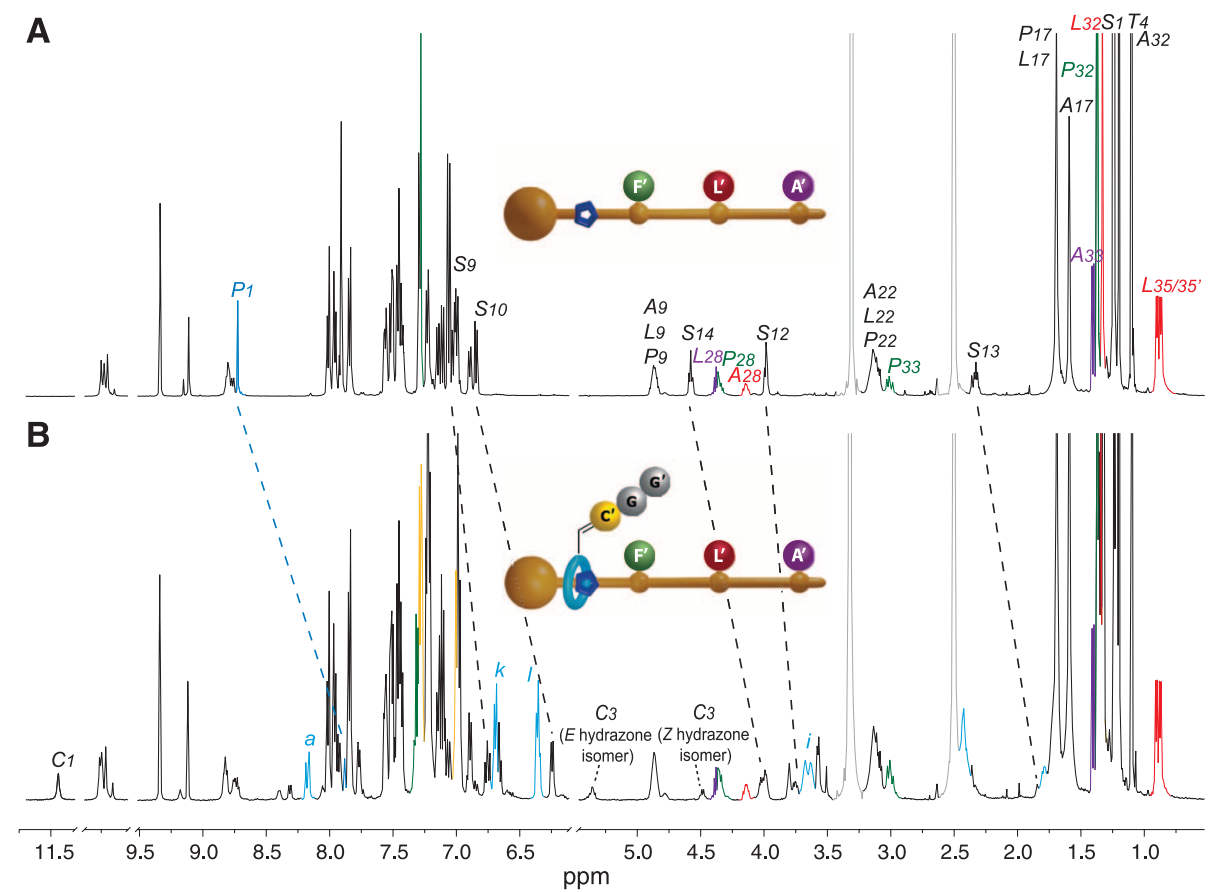


sequence, through chemistry related to nonribosomal peptide synthesis (24).

The chemical structure of the artificial molecular machine, 1, is shown in Fig. 1. Strand 2 bears three amino acids attached to the track by weak phenolic ester linkages (25) and separated from each other by rigid spacers that minimize the possibility of the reactive arm of the machine coming into contact and reacting with a building block out of sequence (26). Macrocycle 3 contains an endotopic pyridine group that directs the threading of strand $\mathbf{2}$ during the $\mathrm{Cu}(\mathrm{I})$-catalyzed cycloaddition of the terminal alkyne with the azide-bearing stopper group 5, leading to the assembly of rotaxane 4 in $30 \%$ yield (Fig. 1, i). This active template $(27,28)$ strategy ensured that the resulting threaded structure did not have residual attractive intercomponent interactions that would tend to localize the position of the ring rather than allow it to move freely up and down the strand between blocking groups. Once we assembled the macrocycle-strand-stopper
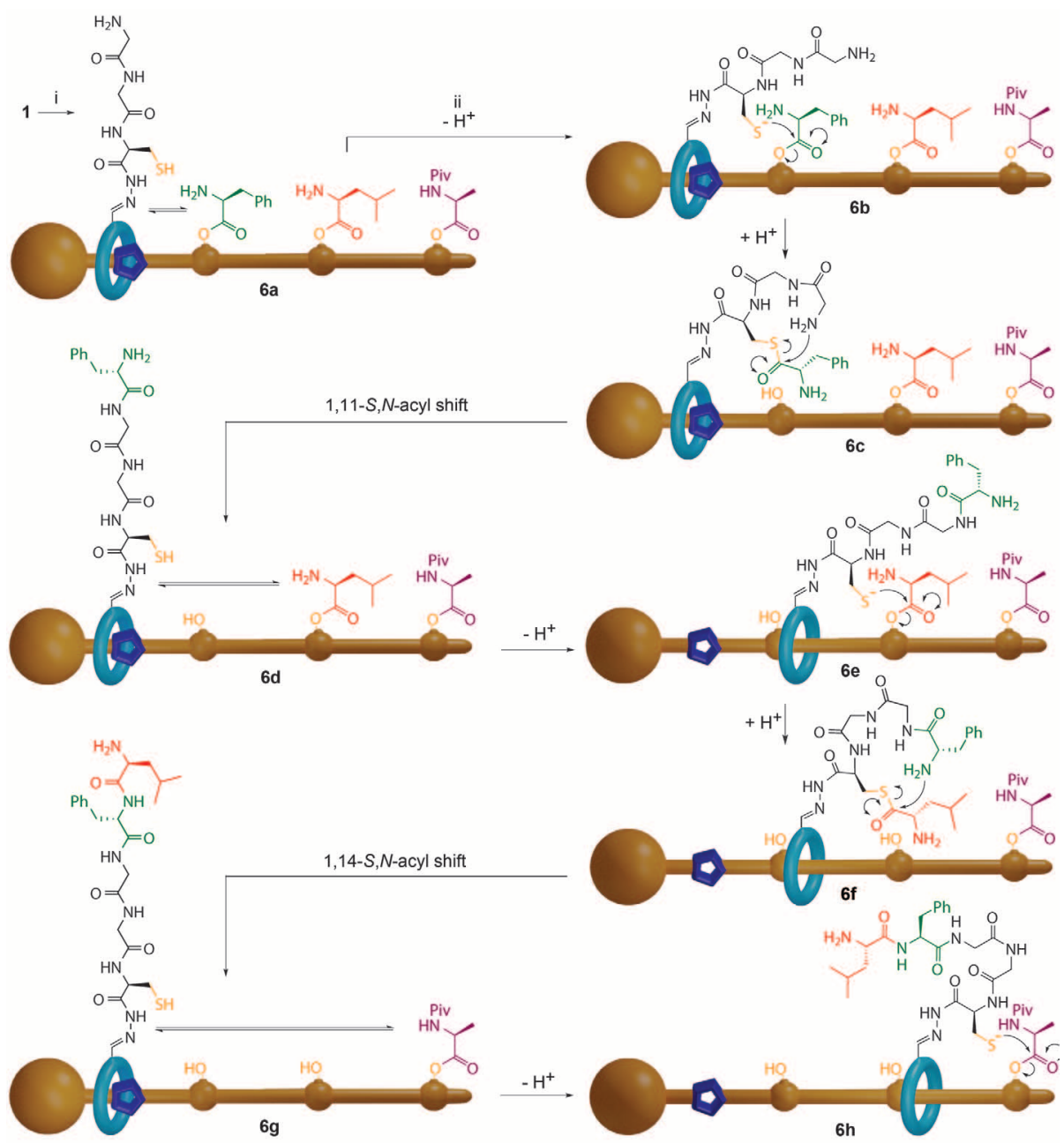

$6 \mathrm{~g}$
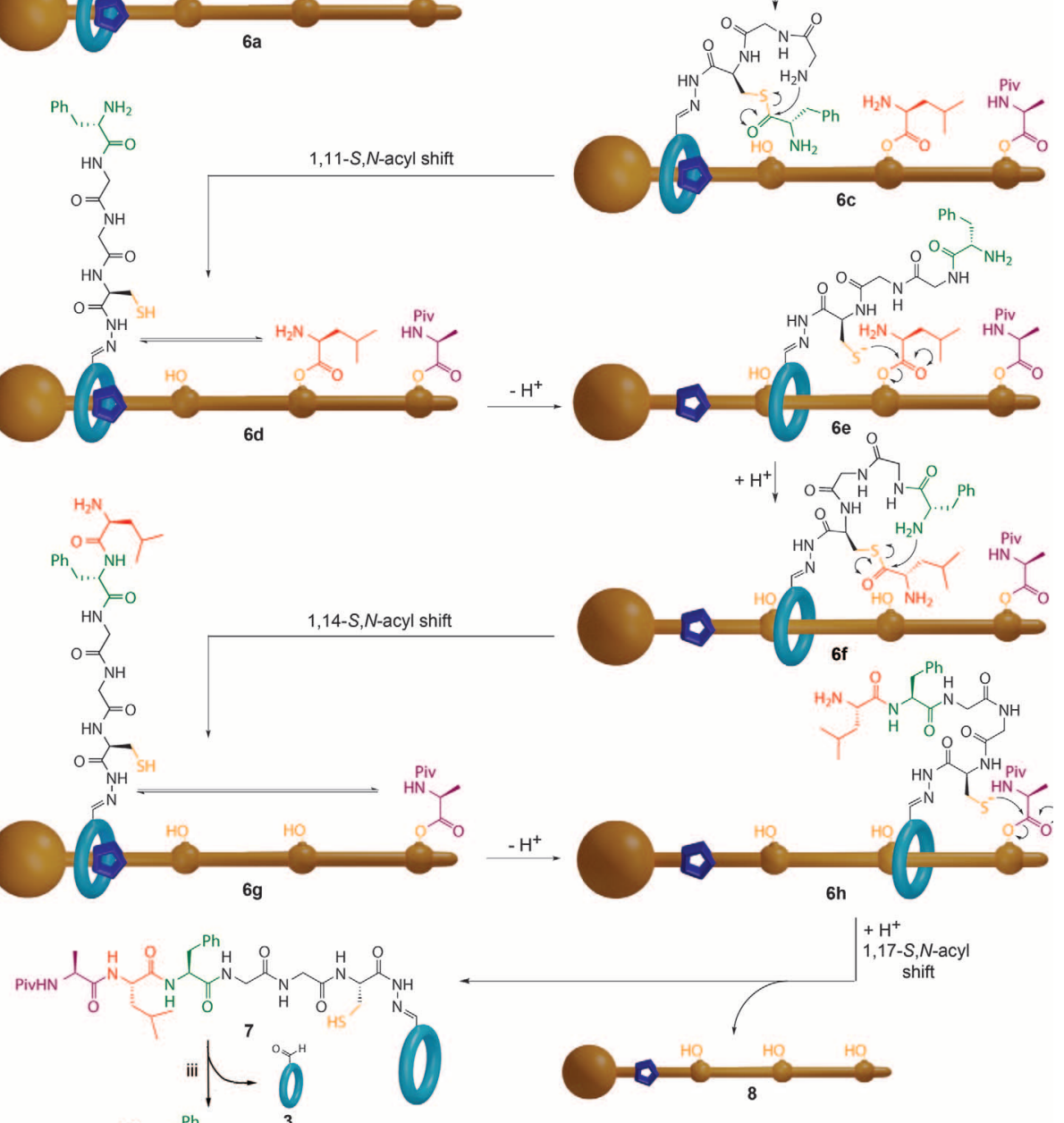

\section{$\mathrm{NH}$}

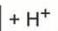

$1,17-S, N$-acyl

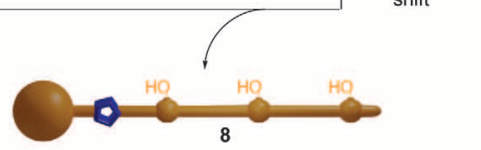

PivHN $\overbrace{O}^{N}$

Fig. 3. Proposed mechanism for sequence-specific peptide synthesis by molecular machine $\mathbf{1}$. After activation of the machine by acidic cleavage of the Boc and Trt protecting groups, under basic conditions successive native chemical ligation reactions transfer the amino acid building blocks to the peptide-elongation site on the macrocycle in the order they appear on the thread. Once the final amino acid is cleaved, the macrocycle bearing the synthesized oligopeptide $\mathbf{7}$ dethreads from the strand. The hydrazide peptide $\mathbf{9}$ is subsequently released from the macrocycle by hydrolysis. Reaction conditions: (i) $20 \% \mathrm{CF}_{3} \mathrm{CO}_{2} \mathrm{H}$ in dichloromethane, room temperature, 2 hours, $100 \%$. (ii) $\left(\left(\mathrm{CH}_{3}\right)_{2} \mathrm{CH}\right)_{2} \mathrm{NEt},\left(\mathrm{HO}_{2} \mathrm{CCH}_{2} \mathrm{CH}_{2}\right)_{3} \mathrm{P}$ in 3:1 acetonitrile:dimethylformamide, $60^{\circ} \mathrm{C}$, 36 hours. Et, ethyl. (iii) $30 \% \mathrm{CF}_{3} \mathrm{CO}_{2} \mathrm{H}$ in 3:1 dichloromethane:water, room temperature, 18 hours. conjugate 4 , we used reversible hydrazone exchange to introduce a cysteine derivative bearing the reactive arm [a trityl (Trt)-protected thiol group] and the site for peptide elongation [a tert-butoxycarbonyl carbamate (Boc)-protected amine at the end of a glycylglycine residue] (Fig. 1, ii). The fully assembled machine $\mathbf{1}$ is stable in its protected form, with upfield shifts of the $\mathrm{H}_{P I}$ triazole and nearby $\mathrm{H}_{S 12-S 14}$ proton signals evident in the ${ }^{1} \mathrm{H}$ nuclear magnetic resonance (NMR) spectrum on account of shielding from the phenyl rings of the macrocycle, confirming that the ring is trapped in the region of the strand between the terminal stopper and the Boc-phenylalanine ester (Fig. 2).

We used acid-catalyzed cleavage of the Boc and trityl protecting groups (Fig. 3, i) to activate the molecular machine and then allowed it to operate (Fig. 3 , ii) at $60^{\circ} \mathrm{C}$ under microwave heating in a 3:1 acetonitrile:dimethylformamide solution in the presence of $N, N$-diisopropylethylamine (a nonnucleophilic base) and tris(2-carboxyethyl)phosphine (a reducing agent that cleaves any disulfide bonds formed through thiol oxidation). The design of the machine is such that once the thiolate residue of the cysteine group (6a) is deprotected, it is poised to undergo a transacylation reaction with the first amino acid phenolic ester that blocks the macrocycle's path on the track $(\mathbf{6 b})$. We hypothesized that the subsequently formed phenylalanine thioester (6c) would be able to react further, transferring the amino acid by native chemical ligation (29) to the glycylglycine amine group by an 11-memberedring transition state [the dipeptide spacer between the cysteine residue and the amine of the peptideelongation site was introduced because native chemical ligation is reported to be very slow via 8-membered-ring transition states (30)]. This sequence simultaneously transfers the amino acid to the end of the growing peptide (6d) and regenerates the catalytic thiolate group, ready for the cleavage and transfer of further building blocks. $S-N$ acyl transfer is a key feature of nonribosomal peptide synthesis (24).

Once the covalent bond connecting an amino acid to the strand is broken, the macrocycle is able to move further along the track until its path is blocked by the next amino acid group (6d). The $O-S$ acyl transfer/ $S-N$ acyl transfer/catalyst regeneration/ring movement process continues (6e to 6h) until the last amino acid on the track is cleaved (6h) and the macrocycle detaches from the strand (8) with the newly formed, full length, peptide attached (7). The artificial molecular machine synthesizes the peptide from the $\mathrm{C}$ terminus to the $\mathrm{N}$ terminus, the opposite direction of ribosomal translation (2-4).

After a 36-hour operation of $\mathbf{6 a}$ at $60^{\circ} \mathrm{C}$, no starting material remained, as shown by highperformance liquid chromatography (HPLC), and two major products were isolated from the reaction mixture (26). We used ${ }^{1} \mathrm{H}$ NMR spectroscopy and mass spectrometry to identify one product as the completely deacylated thread, $\mathbf{8}$. The other product had a ${ }^{1} \mathrm{H}$ NMR spectrum and molecular 
weight (Fig. 4) consistent with the macrocycle bearing the hydrazone linked to the hexapeptide (Piv)AlaLeuPheGlyGlyCys, 7 [Piv, $\mathrm{COC}\left(\mathrm{CH}_{3}\right)_{3}$ ].

To confirm that the product of the molecular machine's operation had the amino acids assembled in the correct order, we used tandem mass spectrometry (MS/MS) to determine the peptide sequence. We then validated the sequence by comparing it with an authentic sample and an isomer in which the order of the Phe and Leu residues was reversed, each prepared unambiguously by conventional peptide synthesis (26). Figure 4A shows the ${ }^{1} \mathrm{H}$ NMR spectrum of the molecular machine product 7 , and Fig. 4B shows the MS/MS spectrum of one isotope of a $2+$ ion of 7 derivatized through the cysteine as an $S, N$-acetal, a species that gave a sufficient signal-to-noise ratio for the MS/MS experiment. Figure 4C shows the superimposition of the MS/MS spectra of a similar isotope and ion from the authentic samples of the macrocycle bearing the sequence (Piv)AlaPheLeuGlyGlyCys (red peaks) and (Piv)AlaLeuPheGlyGlyCys (blue peaks). The difference in the fragmentation masses of the two sequence isomers is apparent in Fig. 4C (725.73 for the LeuGlyGlyCysmacrocycle, 742.92 for the PheGlyGlyCysmacrocycle), and product 7 was confirmed as corresponding to the intended sequence isomer.

With the use of HPLC-MS analysis of the reaction mixture from the operation of $\mathbf{1}$, we did not detect any products corresponding to other peptide compositions (neither different sequences nor peptides with more or less than one Phe, Leu, or Ala residue), indicating that the peptide synthesis occurs overwhelmingly within the confines of the molecular machine. In contrast, a control reaction carried out under identical conditions but using the nonthreaded strand and macrocycle yielded several products, including strands with one or more amino acid groups cleaved, but there was no evidence for the formation of 7 under these conditions. Thus, the threaded architecture of the molecular machine-encompassing the catalytic site, elongation site, and the building block strand - is essential for the sequential peptide synthesis, and the mode of operation of the molecular machine is consistent with the mechanism shown in Fig. 3. The peptide (9), still bearing the GlyGlyCys unit at the $\mathrm{C}$ terminus, could subsequently be cleaved from the macrocycle by hydrolysis (Fig. 3, iii)

On a scale of tens of milligrams, we performed the synthesis of the small peptide through autonomous multistep production by artificial smallmolecule machine $\mathbf{1}$, corresponding to parallel synthesis by $\sim 10^{18}$ machines. Once operation is initiated, the synthetic tasks performed by 1 proceed automatically, requiring no further intervention. As the catalytic thiolate is constrained by the threaded architecture of the machine from reacting with building blocks out of sequence, the act of balancing the rate of reactions with the speed that templates rearrange (19-22) is unnecessary for a rotaxane-based machine.

Rotaxane $\mathbf{1}$ is a (very) primitive analog of the ribosome. Limitations of the first-generation artificial system include slow kinetics (1 takes $\sim 12$ hours to make each amide bond, compared to the 15 to 20 amide bonds synthesized per second by a ribosome) and loss of the sequence information on the strand as it is translated into the product. Furthermore, the size of oligopeptide that can be produced may ultimately be restricted by the size of the cyclic transition states involved

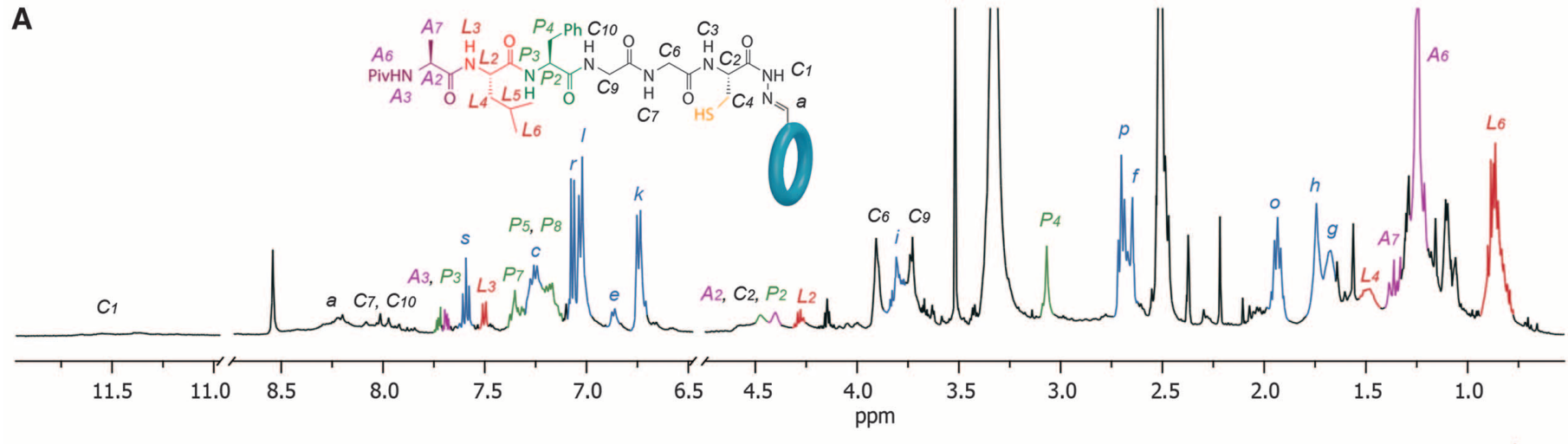

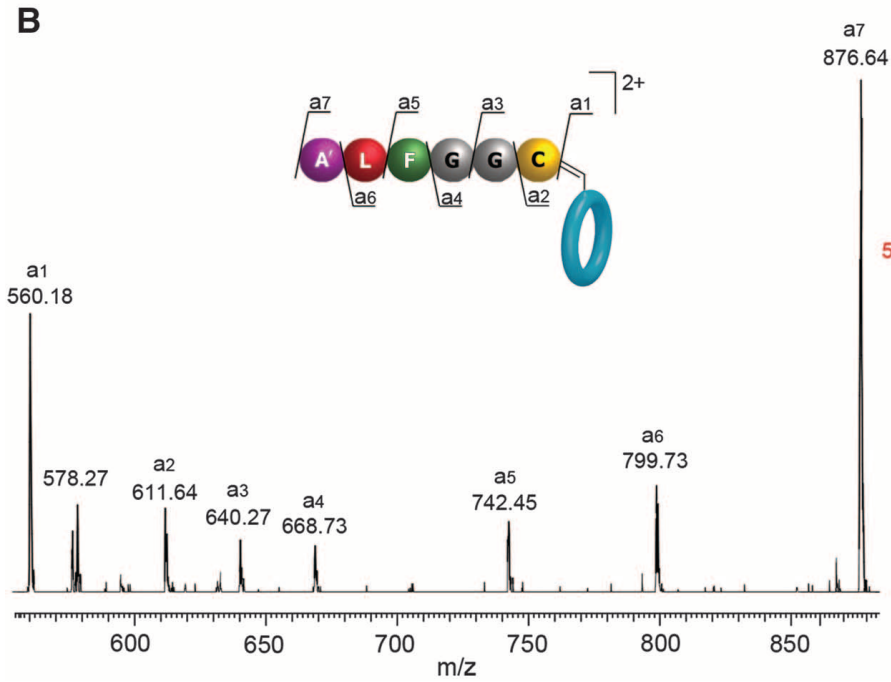

Fig. 4. (A) Proton NMR spectrum of molecular machine operation product 7 in $d_{6}$-dimethylsulfoxide ( $500 \mathrm{MHz}, 298 \mathrm{~K}$ ). (B) Tandem mass spectrum of a single isotope of the $2+$ ion [mass/charge ratio $(\mathrm{m} / \mathrm{z})=876.64$ ] of $S, N$-acetalderivatized 7. (C) Superimposed tandem mass spectra of $2+$ ions of $S, N$-acetal-

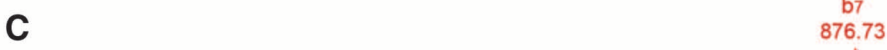

C

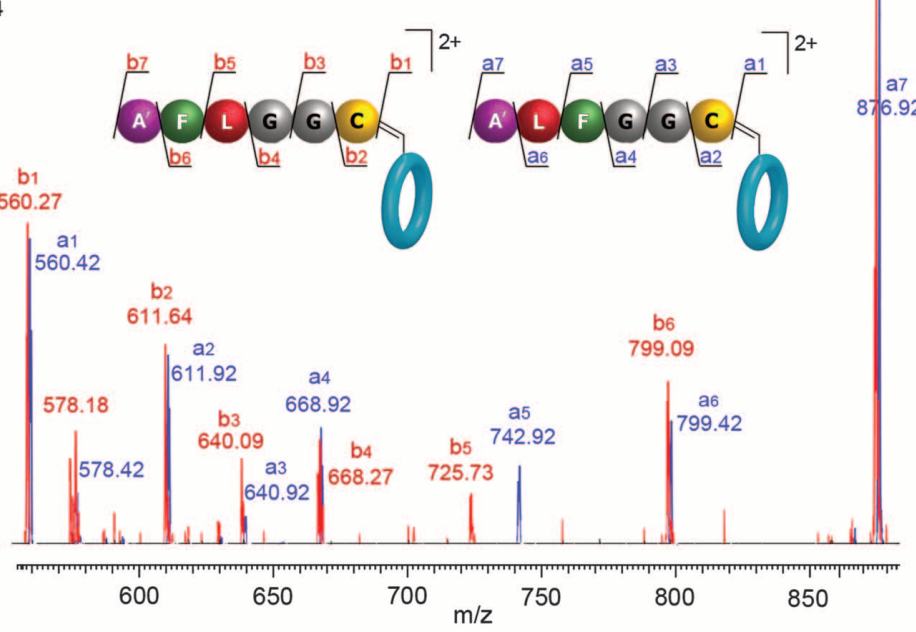

derivatized macrocycles bearing the peptide sequences (Piv)AlaPheLeuGlyGlyCys (red; $2+$ ion isotope-selected $m / z=876.73$ ) and (Piv)AlaLeuPheGlyGlyCys (blue; $2+$ ion isotope-selected $m / z=876.92$ ), each prepared unambiguously by conventional peptide synthesis. 
in $S$-to- $N$ acyl transfer [although peptide ligation has been successfully used with up to 29-membered cyclic transition states (31)]. Nevertheless, 1 demonstrates that relatively small, highly modular, artificial molecular machines can be designed to autonomously perform iterative tasks in synthesis. The principles employed in the design and operation of $\mathbf{1}$ should be broadly applicable to other types of monomer and chemical reactions (32).

\section{References and Notes}

1. ]. M. Berg, ]. L. Tymoczko, L. Stryer, Biochemistry (W. H. Freeman, New York, ed. 6, 2006).

2. A. Yonath, Angew. Chem. Int. Ed. 49, 4340 (2010).

3. V. Ramakrishnan, Angew. Chem. Int. Ed. 49, 4355 (2010).

4. T. A. Steitz, Angew. Chem. Int. Ed. 49, 4381 (2010).

5. E. R. Kay, D. A. Leigh, F. Zerbetto, Angew. Chem. Int. Ed. 46, 72 (2007).

6. C. P. Collier et al., Science 285, 391 (1999).

7. J. E. Green et al., Nature 445, 414 (2007).

8. J. Berná et al., Nat. Mater. 4, 704 (2005).

9. R. Eelkema et al., Nature 440, 163 (2006).

10. T. Muraoka, K. Kinbara, T. Aida, Nature 440, 512 (2006).

11. B. K. Juluri et al., ACS Nano 3, 291 (2009).

12. P. Thordarson, E. J. Bijsterveld, A. E. Rowan,

R. ]. M. Nolte, Nature 424, 915 (2003).
13. C. Monnereau et al., J. Am. Chem. Soc. 132, 1529 (2010).

14. F. Würthner, ]. Rebek Jr., Angew. Chem. Int. Ed. Engl. 34, 446 (1995).

15. H. J. Yoon, ]. Kuwabara, J.-H. Kim, C. A. Mirkin, Science 330, 66 (2010).

16. M. Schmittel, S. De, S. Pramanik, Angew. Chem. Int. Ed. 51, 3832 (2012)

17. V. Blanco, A. Carlone, K. D. Hänni, D. A. Leigh, B. Lewandowski, Angew. Chem. Int. Ed. 51, 5166 (2012).

18. J. Wang, B. L. Feringa, Science 331, 1429 (2011).

19. Y. He, D. R. Liu, Nat. Nanotechnol. 5, 778 (2010).

20. M. L. McKee et al., Angew. Chem. Int. Ed. 49, 7948 (2010).

21. M. L. McKee et al., J. Am. Chem. Soc. 134, 1446 (2012).

22. Y. He, D. R. Liu, J. Am. Chem. Soc. 133, 9972 (2011).

23. H. Gu, J. Chao, S.-J. Xiao, N. C. Seeman, Nature 465, 202 (2010).

24. S. A. Sieber, M. A. Marahiel, Chem. Rev. 105, 715 (2005).

25. S,S-acyl transfer is readily reversible, so employing thioesters (the most commonly used acyl source for native chemical ligation) to attach the building blocks to the strand would risk an amino acid being returned to the track after the macrocycle had passed by, potentially reducing the sequence integrity of the peptide synthesis.

26. Materials and methods are available as supplementary materials on Science Online.

27. V. Aucagne, K. D. Hänni, D. A. Leigh, P. J. Lusby, D. B. Walker, J. Am. Chem. Soc. 128, 2186 (2006).
28. J. D. Crowley, S. M. Goldup, A.-L. Lee, D. A. Leigh, R. T. McBurney, Chem. Soc. Rev. 38, 1530 (2009).

29. P. E. Dawson, T. W. Muir, I. Clark-Lewis, S. B. Kent, Science 266, 776 (1994).

30. F. K. Hansen et al., Org. Biomol. Chem. 9, 7162 (2011)

31. R. J. Payne et al., J. Am. Chem. Soc. 129, 13527 (2007).

32. N. Badi, J.-F. Lutz, Chem. Soc. Rev. 38, 3383 (2009).

Acknowledgments: We thank V. Aucagne for useful suggestions during early versions of the molecular machine design. This research was funded by the Engineering and Physical Sciences Research Council (UK). We are grateful to the following organizations for postdoctoral fellowships: Fundacja na Rzecz Nauki Polskiej (to B.L.), Fonds de la Recherche Scientifique and Wallonie-Bruxelles International (to G.D.B.), the European Union 7th Framework Marie Curie Intra European Fellowship Program (to M.J.A.), Deutscher Akademischer Austausch Dienst (to P.M.E.G. and D.H.), and Deutsche Akademia der Naturforscher Leopoldina and Peter und Traudl Engelhorn-Stiftung (to D.M.D.).

\section{Supplementary Materials}

www.sciencemag.org/cgi/content/full/339/6116/189/DC1

Materials and Methods

Supplementary Text

Figs. S1 to $\mathrm{S37}$

References (33-35)

5 September 2012; accepted 20 November 2012 $10.1126 /$ science. 1229753

\section{Shape-Memory Nanopores Induced in Coordination Frameworks by Crystal Downsizing}

\section{Yoko Sakata, ${ }^{1,2}$ Shuhei Furukawa, ${ }^{1,2 *}$ Mio Kondo, ${ }^{1,2}$ Kenji Hirai, $^{3}$ Nao Horike, ${ }^{2}$ Yohei Takashima, ${ }^{2}$ Hiromitsu Uehara, ${ }^{2}$ Nicolas Louvain, ${ }^{1,2}$ Mikhail Meilikhov, ${ }^{2}$ Takaaki Tsuruoka, ${ }^{2,5}$ Seiji Isoda, ${ }^{1}$ Wataru Kosaka, ${ }^{2}$ Osami Sakata, ${ }^{4}$ Susumu Kitagawa ${ }^{1,2,3 *}$}

Flexible porous coordination polymers change their structure in response to molecular incorporation but recover their original configuration after the guest has been removed. We demonstrated that the crystal downsizing of twofold interpenetrated frameworks of $\left[\mathrm{Cu}_{2} \text { (dicarboxylate) }{ }_{2} \text { (amine) }\right]_{n}$ regulates the structural flexibility and induces a shape-memory effect in the coordination frameworks. In addition to the two structures that contribute to the sorption process (that is, a nonporous closed phase and a guest-included open phase), we isolated an unusual, metastable open dried phase when downsizing the crystals to the mesoscale, and the closed phase was recovered by thermal treatment. Crystal downsizing suppressed the structural mobility and stabilized the open dried phase. The successful isolation of two interconvertible empty phases, the closed phase and the open dried phase, provided switchable sorption properties with or without gate-opening behavior.

$\mathrm{S}$ hape-memory materials alter their morphological appearance in response to an external stimulus (for example, mechanical stress created by macroscopic structural deformation), hold their new temporary shape after the stimulus has been removed, and return to their original morphology in the presence of another external stimulus $(1,2)$. For instance, a metal alloy can exhibit shape-memory effect if it has two phases that can interconvert reversibly; that is, without requiring atoms to diffuse through the structure. Here, we describe a molecular-scale shape-memory effect (MSME) in nanoporous framework materials in which the application of an adsorption stress deforms the original shape of the nanopore into a temporary shape, which is maintained even after desorption, and thermal treatment then recovers the original shape.

Our design takes advantage of the flexibility of crystalline porous coordination polymers (PCPs) (3-8), which are assembled from organic spokes and inorganic joints. These flexible PCPs cooperatively reconfigure their framework structures in response to the incorporation of molecules into the nanopores; this adsorption process triggers the deformation of the pore shape. Most flexible
PCPs recover the original structure after the removal of the adsorption stress (that is, the desorption of the guest molecules), which leads to the so-called framework elasticity property (9). The MSME requires that any structural transformation during desorption should be suppressed. We show that crystal downsizing influences the structural mobility, because a reduction in the number of repeating units should be sufficient to regulate the cooperative nature of the structural transformation and the effect of stress.

We fabricated MSME nanopores by crystal downsizing, which regulated the flexibility of the framework, and demonstrated the switchable sorption events based on the presence of two interconvertible pore shapes (Fig. 1). Among the variety of flexible PCPs, we chose a PCP with a twofold interpenetrated framework (10-12)-namely, $\left[\mathrm{Cu}_{2}(\mathrm{bdc})_{2}(\mathrm{bpy})\right]_{n}(\mathbf{1}, \mathrm{bdc}=1,4$-benzenedicarboxylate, bpy $=4,4$ '-bipyridine) (13) - that exhibits a cooperative structural transformation from the

${ }^{1}$ World Premier International Research Initiative-Institute for Integrated Cell-Material Sciences (WPI-iCeMS), Kyoto University, Yoshida, Sakyo-ku, Kyoto 606-8501, Japan. 'Exploratory Research for Advanced Technology (ERATO) Kitagawa Integrated Pores Project, Japan Science and Technology Agency (JST), Kyoto Research Park Building \#3, Shimogyo-ku, Kyoto 600-8815, Japan. ${ }^{3}$ Department of Synthetic Chemistry and Biological Chemistry, Graduate School of Engineering, Kyoto University, Katsura, Nishikyo-ku, Kyoto 615-8510, Japan. ${ }^{4}$ Synchrotron X-ray Station at SPring-8, National Institute for Materials Science (NIMS), Kouto, Sayo, Hyogo 679-5148, Japan. ${ }^{5}$ Frontiers of Innovative Research in Science and Technology (FIRST), Konan University, 7-1-20, Minatojima-minamimachi, Chuo-ku, Kobe 650-0047, Japan.

*To whom correspondence should be addressed. E-mail: shuhei.furukawa@icems.kyoto-u.ac.jp (S.F.); kitagawa@ icems.kyoto-u.ac.jp (S.K.) 